\title{
La tasa social de descuento en la evaluación de proyectos de inversión: una aplicación para el Ecuador ${ }^{1}$
}

\author{
José Gabriel Castillo y Donald Zhangallimbay
}

\section{Resumen}

La tasa social de descuento estándar aplicada por instituciones de planificación y organismos multilaterales (12\%) en la evaluación de proyectos públicos es un parámetro administrativo, constante, sin respaldo en su determinación y que no reconoce los cambios de las preferencias sociales en el tiempo. En este trabajo presentamos una alternativa de determinación de la tasa social de descuento basada en el modelo de estimación gamma (Weitzman, 2001), en el contexto de países en desarrollo, que posee tres ventajas: i) incorpora el descuento decreciente; ii) es eficiente en relación con el costo, por cuanto resume las diversas opiniones de expertos, y iii) se ajusta a los cambios en las preferencias de corto y largo plazo. En nuestras estimaciones se obtienen niveles inferiores a la tasa nominal estándar, para distintos períodos de tiempo, que fluctúan entre un $2 \%$, para horizontes de evaluación superiores a 51 años, y un 11\%, para el corto plazo (de 0 a 5 años).

\section{Palabras clave}

Inversiones, proyectos de desarrollo, preparación de proyectos, evaluación de proyectos, igualdad, economía del bienestar, modelos matemáticos, países en desarrollo, Ecuador

\section{Clasificación JEL}

O22, D04, C93

\section{Autores}

José Gabriel Castillo es Director del Centro de Investigaciones Económicas (CIEC) y del Laboratorio de Economía Experimental y del Comportamiento (LEE) de la Facultad de Ciencias Sociales y Humanísticas (FCSH) de la Escuela Superior Politécnica del Litoral (ESPOL) del Ecuador. Correo electrónico: jcastil@espol.edu.ec.

Donald Zhangallimbay es Coordinador e Investigador del Laboratorio de Economía Experimental y del Comportamiento (LEE) de la Facultad de Ciencias Sociales y Humanísticas (FCSH) de la Escuela Superior Politécnica del Litoral (ESPOL) del Ecuador. Correo electrónico: djzhanga@espol.edu.ec.

\footnotetext{
El estudio es financiado por fondos de investigación de proyectos del Centro de Investigaciones Económicas (CIEC) y la Facultad de Ciencias Sociales y Humanísticas (FCSH) de la Escuela Superior Politécnica del Litoral (ESPOL) del Ecuador. Esta investigación es un producto que se deriva del proyecto "La tasa social de descuento en el Ecuador: una mirada desde la economía experimental" (CIEC-19-2016).
} 


\section{Introducción}

La evaluación de costo-beneficio es la principal herramienta empleada para determinar la factibilidad económica de proyectos de inversión pública. La tasa social de descuento (TSD) es un parámetro fundamental de este análisis, particularmente porque representa el costo de oportunidad del uso de los recursos en el tiempo y permite comparar los ingresos y los costos (flujos de fondos netos) proyectados en distintos períodos. En este contexto, una TSD demasiado elevada puede significar el rechazo de proyectos socialmente deseables; de modo opuesto, una TSD demasiado baja puede redundar en el desperdicio de recursos en proyectos económicamente ineficientes (Zhuang y otros, 2007). La elección de una tasa de descuento apropiada no es un ejercicio trivial; por el contrario, es un tema de intenso debate en la literatura económica y financiera, particularmente en lo que respecta al planteamiento de metodologías y estrategias eficientes que garanticen la optimización del uso de los recursos y el desarrollo social sostenible (véase un resumen en Correa, 2008a).

Hasta la fecha no existe un consenso sobre cómo medir y establecer una adecuada tasa social de descuento (Campos, Serebrisky y Suárez-Alemán, 2015). Sin embargo, debido a la creciente exigencia social de transparencia administrativa y al intenso debate sobre las externalidades de los proyectos reflejadas en la contaminación y sus efectos en el medio ambiente, diversos países han emprendido la revisión de los parámetros de evaluación económica empleados. Las economías de América Latina y el Caribe (por ejemplo, Chile, Colombia y Costa Rica) no son la excepción. Los notables avances de la región en cuanto a desarrollo económico y acceso a financiamiento externo plantean la necesidad de reevaluar la TSD como instrumento de análisis de la eficiencia y la equidad intergeneracional de la inversión pública (Campos, Serebrisky y Suárez-Alemán, 2015).

Existen tres enfoques comúnmente aplicados para determinar la TSD: i) el enfoque de la tasa social de preferencia intertemporal, ii) el enfoque de costo de oportunidad social del capital y iii) el enfoque combinado, de promedio ponderado o de Harberger (Harberger, 1972). Sin embargo, las técnicas tradicionales enmarcadas en un modelo de tasa de descuento constante son ineficientes cuando se intenta evaluar proyectos con un elevado componente de externalidad no absorbido por el mercado, o proyectos con efectos intergeneracionales - o con efectos a muy largo plazo-, por ejemplo, aquellos de infraestructura de transporte, construcción de hospitales, construcción de carreteras o eliminación de residuos, entre otros. La búsqueda de parámetros compatibles con las preferencias sociales motiva a reconsiderar los efectos de la inversión pública, más aún cuando el efecto se extiende social y económicamente a futuras generaciones, que no intervienen en la decisión de inversión (Correa, 2008b). Las tasas que actualmente se aplican en varios países en desarrollo, por ejemplo en el Ecuador, omiten varios criterios de evaluación técnica y sugieren un nivel relativamente elevado de descuento, lo que caracteriza la preferencia del uso de los recursos en períodos de corto plazo y devalúa rápidamente los beneficios económicos que se obtendrán en el futuro lejano (Frederick, Loewenstein y O’Donoghue, 2002).

Si bien una TSD constante facilita la modelización e interpretación de los flujos económicos, una vasta literatura empírica y experimental tanto del ámbito de la psicología como de la economía (véanse, por ejemplo, Thaler, 1981; Harrison y otros, 2005; Benhabib, Bisin y Schotter, 2010; Castillo y Zhangallimbay, 2018) expone múltiples anomalías de las preferencias sobre el tiempo, que favorecen una modalidad de tasas de descuento decrecientes; es decir, a medida que el plazo de espera en la recepción de flujos monetarios aumenta, los individuos tienden a ser más pacientes, lo que se traduce en tasas de descuento más bajas. Esta anomalía del comportamiento ya ha sido considerada en modalidades de evaluación de proyectos de inversión. En el Reino Unido, por ejemplo, el método estándar para descontar flujos de largo plazo (de más de 30 años) emplea tasas decrecientes que van del 3,5\% al 1,0\% (Lowe, 2008). 
Por otro lado, Delacámara (2008) recomienda el uso de una tasa social de descuento que incorpore las preferencias sociales sobre el tiempo. En el Ecuador, al igual que en otros países de América Latina, se emplea una tasa fija de descuento, por lo general del 12\%. Esta tasa no se origina en una estimación de las preferencias individuales o sociales sobre el tiempo, sino que, por el contrario, constituye una tasa administrativa sugerida tanto por organismos internacionales (por ejemplo, el Banco Interamericano de Desarrollo (BID) y el Banco Mundial) como por instituciones nacionales de planificación. Varios países han emprendido un proceso de revisión de su TSD con resultados interesantes; las estimaciones resultantes son, por lo general, menores que el nivel de descuento aplicado. Solo en el Ecuador la Secretaría Nacional de Planificación y Desarrollo (SENPLADES) evalúa aproximadamente 140 proyectos por año empleando la TSD, y en ninguno de esos casos se realiza una diferenciación en términos de si el período de ejecución es de corto o largo plazo.

En este estudio revisamos la estimación de la TSD diferenciando cuatro períodos de evaluación entre el corto y el largo plazo: futuro inmediato (1 a 5 años), futuro mediano (6 a 20 años), futuro lejano (21 a 50 años) y futuro muy lejano (51 a 100 años). En nuestras estimaciones para un país en desarrollo, el Ecuador, empleamos el mecanismo de descuento gamma, propuesto por Weitzman (2001), para integrar la opinión de un grupo diverso de responsables de la toma de decisiones, adecuando un formulario de modo que permita adaptar la estimación de intervalos para afinar los resultados (Edwards, 2016). Adicionalmente, presentamos un ajuste en el que consideramos el sesgo de valor presente generado por las preferencias sobre el tiempo en un contexto de corto plazo. Los resultados muestran que las tasas determinadas en este contexto varían respecto de las estimadas mediante el mecanismo de encuesta general de largo plazo. Promediando los resultados obtenidos por ambos procedimientos, observamos un ajuste significativo de la tasa recomendada para el futuro inmediato ( 1 a 5 años), lo que representa un argumento de relevancia económica frente al uso de la tasa estándar. Asimismo, los resultados sugieren el uso de una tasa del 11\% para el futuro inmediato, del $4 \%$ para el futuro mediano, del $3 \%$ para el futuro lejano y del $2 \%$ para el futuro muy lejano (para períodos superiores a 100 años, se recomienda emplear el 1\%). Finalmente, analizamos los factores relacionados con las preferencias de los entrevistados en lo referente a sus tasas sociales de descuento, y observamos que tanto la edad como los niveles de aversión al riesgo son condiciones relevantes en la conformación de las preferencias sobre el tiempo.

El resto del artículo se organiza en cuatro secciones. En la segunda sección se presenta una revisión de las metodologías y los resultados obtenidos en la reestimación de las TSD en diversos países. En la tercera sección se detallan la metodología, los datos y el ajuste aplicado para corregir los sesgos de exposición de los formularios tradicionales. En la cuarta sección se presentan los resultados del estudio y una discusión de sus implicaciones. Finalmente, en la quinta sección se exponen las conclusiones del trabajo.

\section{Alternativas metodológicas y experiencias internacionales}

Ante la ausencia de un consenso sobre la definición y los mecanismos de estimación de la tasa social de descuento, es posible encontrar diversas maneras de aproximar este parámetro. Las estimaciones realizadas en varios países del mundo (véase el cuadro 1) muestran que el supuesto de una tasa constante derivada del descuento exponencial sigue siendo el método más aplicado (Campos, Serebrisky y Suárez-Alemán, 2015). 
Cuadro 1

Estimaciones de la tasa social de descuento en el mundo

\begin{tabular}{|c|c|c|}
\hline Institución o país & Tasa & Base conceptual o teórica \\
\hline \multicolumn{3}{|c|}{ Organismos multilaterales } \\
\hline Banco Mundial & $10 \%-12 \%$ & Tasa administrativa convencional \\
\hline Banco Interamericano de Desarrollo & $10 \%-12 \%$ & $\begin{array}{l}\text { Tasa administrativa convencional/ } \\
\text { costo de oportunidad del capital }\end{array}$ \\
\hline Banco Asiático de Desarrollo & $10 \%-12 \%$ & Tasa administrativa convencional \\
\hline \multicolumn{3}{|l|}{ Países desarrollados } \\
\hline Alemania & $3 \%$ & Basada en la tasa federal de refinanciamiento \\
\hline Canadá & $10 \%$ & Costo de oportunidad social del capital \\
\hline España & $\begin{array}{l}6 \% \text { para transporte } \\
4 \% \text { para agua }\end{array}$ & Tasa social de preferencia intertemporal \\
\hline $\begin{array}{l}\text { Estados Unidos, Oficina de } \\
\text { Administración y Presupuesto }\end{array}$ & $7 \%$ & Costo de oportunidad social del capital \\
\hline $\begin{array}{l}\text { Estados Unidos, Oficina de } \\
\text { Presupuesto del Congreso y } \\
\text { Oficina General de Contabilidad }\end{array}$ & Tasa de mercado de deuda del Tesoro & Tasa social de preferencia intertemporal \\
\hline $\begin{array}{l}\text { Estados Unidos, Agencia de } \\
\text { Protección Ambiental }\end{array}$ & $\begin{array}{l}\text { Tasa intergeneracional de descuento: } \\
2 \%-3 \% \text {, sujeta a análisis de sensibilidad }\end{array}$ & Tasa social de preferencia intertemporal \\
\hline Francia & $4 \%$ & Tasa social de preferencia intertemporal \\
\hline Noruega & $3,5 \%$ & Tasa de crédito gubernamental en términos reales \\
\hline Reino Unido & $\begin{array}{l}3,5 \% \\
\text { Para proyectos superiores a } 30 \text { años } \\
\text { tasas diferenciadas menores }\end{array}$ & Tasa social de preferencia intertemporal \\
\hline \multicolumn{3}{|c|}{ Países en desarrollo } \\
\hline China & $\begin{array}{l}8 \% \text { para proyectos de corto y } \\
\text { mediano plazo; menor que } 8 \% \\
\text { para proyectos de largo plazo }\end{array}$ & $\begin{array}{l}\text { Promedio ponderado de la tasa social de preferencia } \\
\text { intertemporal y la tasa basada en el costo de } \\
\text { oportunidad social del capital (método de Harberger) }\end{array}$ \\
\hline India & $12 \%$ & Costo de oportunidad social del capital \\
\hline Pakistán & $12 \%$ & Costo de oportunidad social del capital \\
\hline \multicolumn{3}{|l|}{ Países de América Latina } \\
\hline Chile & $6 \%$ & $\begin{array}{l}\text { Promedio ponderado de la tasa social de preferencia } \\
\text { intertemporal y la tasa basada en el costo de } \\
\text { oportunidad social del capital (método de Harberger) }\end{array}$ \\
\hline Colombia & $12 \%$ & $\begin{array}{l}\text { Equivale a la rentabilidad mínima } \\
\text { esperada por el inversionista }\end{array}$ \\
\hline México & $\begin{array}{l}10 \% \\
\text { antes de } 2014: 12 \%\end{array}$ & $\begin{array}{l}\text { Promedio ponderado de la tasa social de preferencia } \\
\text { intertemporal y la tasa basada en el costo de } \\
\text { oportunidad social del capital (método de Harberger) }\end{array}$ \\
\hline Perú & $\begin{array}{l}9 \% \\
\text { antes de 2012: 10\% }\end{array}$ & $\begin{array}{l}\text { Promedio ponderado de la tasa social de preferencia } \\
\text { intertemporal y la tasa basada en el costo de } \\
\text { oportunidad social del capital (método de Harberger) }\end{array}$ \\
\hline
\end{tabular}

Fuente: J. Campos, T. Serebrisky y A. Suárez-Alemán, Tasa de descuento social y evaluación de proyectos: algunas reflexiones prácticas para América Latina y el Caribe, Banco Interamericano de Desarrollo (BID), 2016; J. Zhuang y otros, "Theory and practice in the choice of social discount rate for cost-benefit analysis: a survey", ERD Working Paper, NN 94, Banco Asiático de Desarrollo, 2007; E. Aldunate y R. Martner, "Política fiscal y protección social", Revista de la CEPAL, № 90 (LC/G.2323-P), Santiago, Comisión Económica para América Latina y el Caribe (CEPAL), 2006; Chile: Sistema Nacional de Inversiones (SNI) del Ministerio de Desarrollo Social; Colombia: Sistema Nacional de Inversión Pública del Departamento Nacional de Planeación; México: Sistema de Inversión Pública (SIP) de la Secretaría de Hacienda y Crédito Público; Perú: Sistema Nacional de Inversión Pública (SNIP) de la Dirección General de Inversión Pública del Ministerio de Economía y Finanzas.

La literatura económica y financiera agrupa los diversos mecanismos de estimación de la TSD en tres grandes enfoques. El primero parte del punto de vista del consumidor; el descuento se deriva de las preferencias sociales por el consumo presente (impaciencia), las expectativas de consumo futuro y las preferencias puras sobre el tiempo (Dasgupta y Pearce, 1972). Este método se conoce como tasa social de preferencia intertemporal. Desde este enfoque, Zhuang y otros (2007) proponen la estimación de la TSD de dos maneras: i) a partir de la rentabilidad de los bonos de gobierno u otro instrumento de bajo riesgo (hasta ahora no se han desarrollado mercados con períodos superiores a 20 años) y ii) a través de la estimación de los distintos componentes de la ecuación de Ramsey (1928): 


$$
r_{t}=\delta+\eta\left(C_{t}\right) \frac{d C_{t}}{C_{t}}
$$

En la mayoría de los casos observados, las estimaciones convergen a esta segunda alternativa, a partir de estimaciones macroeconómicas de los parámetros de la ecuación (1). La tasa social de descuento $r_{t}$ es el resultado de la agregación de dos elementos: i) las preferencias puras sobre el tiempo $(\delta)$ y ii) el producto de dos factores: la elasticidad intertemporal del consumo $\left(\eta\left(C_{t}\right)\right)$ y la tasa anual de crecimiento real del consumo per cápita $\left(\frac{d C_{t}}{C}\right)$. Empleando este método, Zhuang y otros (2007), por ejemplo, estimaron la TSD para varios países de Asia, entre ellos Indonesia, el Japón y Singapur, para los cuales obtuvieron tasas del 6,1\%, el 4,5\% y el 7,3\%, respectivamente. La Comisión Europea (2008) también determina los niveles de descuento social para varios de sus países miembros. En los casos de Alemania, Dinamarca, Francia e Italia, los resultados muestran tasas del 3,1\%, el 3,5\%, el 3,4\% y el $3,3 \%$, respectivamente. Una generalidad observada en las experiencias internacionales es que los resultados se encuentran en niveles inferiores a los de la tasa aplicada en varios países en desarrollo como, por ejemplo, Chile, Colombia, la India y el Perú (véase el cuadro 1). En estos países, e incluso en el Ecuador, persiste la necesidad de realizar una evaluación más exhaustiva de la TSD que considere los cambios en las condiciones de bienestar derivados de los procesos históricos de inversión pública.

El segundo enfoque de estimación de la TSD toma como punto de partida la perspectiva del productor (empresa). El principio básico de esta metodología es que el Gobierno invierte de forma directa fondos que de otra forma podrían ser aprovechados por el sector privado y, por lo tanto, las empresas pierden la oportunidad de emplear esos recursos en iniciativas económicamente rentables. En este contexto, la TSD debe mostrar el retorno mínimo del proyecto con el cual se puede cubrir la rentabilidad perdida del sector privado (Edwards, 2016). Este enfoque se conoce como costo de oportunidad social del capital.

En un entorno económico sin distorsiones, la tasa social de descuento estimada desde ambos enfoques (tasa social de preferencia intertemporal o costo de oportunidad social del capital) debería converger hacia la tasa de interés real de la economía. Sin embargo, suponer un mercado sin fallos es poco realista; los subsidios, impuestos y externalidades no absorbidas por el sistema de precios, entre otros, son todos elementos que distorsionan las relaciones económicas. En este contexto, la aplicación de una u otra estrategia mostrará diferencias en los niveles resultantes de la tasa de descuento (Campos, Serebrisky y Suárez-Alemán, 2015). Desde el punto de vista del capital, si se considera la apertura comercial, su libre movilidad internacional y la flexibilidad de los tipos de cambio, la TSD vendría dada por la tasa de interés de endeudamiento externo, debidamente ajustada por el precio esperado de las divisas en el servicio de la deuda (Fontaine, 2000). Una de las limitaciones de este método es que el mercado internacional no ha desarrollado transacciones en períodos de muy largo plazo (mayores que 30 años), lo que impide generar tasas aproximadas a los precios del capital en horizontes lejanos de evaluación. En la práctica, las instituciones de planificación suelen asumir una tasa de descuento constante; es decir, toman las tasas desarrolladas en los mercados existentes (corto plazo) y asumen su equivalencia a las tasas en períodos de largo y muy largo plazo. Esto es una limitación técnica en la evaluación de proyectos públicos que sobreviven de forma intergeneracional, aunque se evalúan únicamente a tasas de mercados con plazos más cortos, por ejemplo, de hasta 20 años.

La Oficina de Administración y Presupuesto de los Estados Unidos y la Secretaría de la Junta del Tesoro del Canadá, aplicando la metodología de costo de oportunidad social del capital, estiman y emplean tasas del $7 \%$ y el 10\%, respectivamente, en la evaluación de costo-beneficio de sus proyectos de inversión pública. Por otro lado, la aplicación de este enfoque en algunos casos arroja tasas relativamente más elevadas que los niveles aplicados; por ejemplo, en Filipinas y el Pakistán se estiman tasas sociales de descuento del $15 \%$ y el $12 \%$, respectivamente. No obstante, en la mayoría de los casos estudiados (véanse algunos ejemplos en Zhuang y otros, 2007), las TSD estimadas son inferiores a la tasa administrativa. 
Un tercer enfoque para estimar la TSD es el de Harberger (1972). Este enfoque considera las preferencias de descuento tanto de los consumidores como de las empresas; el financiamiento proviene de estas fuentes (desplazamiento del consumo y sacrificio de rentabilidad privada). En este contexto, la TSD es un promedio ponderado de la tasa marginal de preferencia intertemporal $(\delta)$ y la tasa marginal del rendimiento del capital $(R)$, incluidos los ajustes respectivos por riesgo e impuestos. En síntesis, la idea propuesta describe una tasa de la siguiente forma: $T S D=\alpha R+(1-\alpha) \delta$, donde $\alpha$ es la proporción de recursos obtenidos a través del desplazamiento de fondos de inversión privada y $(1-\alpha)$ es la proporción de recursos generados por el desplazamiento del consumo presente. Organismos multilaterales como el Banco Mundial y el Banco Interamericano de Desarrollo (BID) recomiendan este método en sus manuales. Países como China, México y el Perú han aplicado esta metodología y han obtenido tasas del $8 \%$, el $10 \%$ y el $9 \%$, respectivamente.

En los últimos años, el desarrollo del conocimiento y la aplicación de la economía experimental y del comportamiento en la identificación de las preferencias individuales, así como el análisis de equidad intergeneracional de los efectos de la inversión pública, han desafiado las formas tradicionales de estimación de la TSD. Las limitaciones son importantes, toda vez que la asignación de recursos sociales está sujeta a la evaluación económica, en que la TSD es un parámetro determinante. Existen varias alternativas de estimación que superan estas limitaciones, entre ellas el uso de mecanismos de tasas variables en el tiempo (generalmente decrecientes) (Weitzman, 2001) o modelos alternativos de descuento, por ejemplo, el modelo hiperbólico (Mazur, 1987). En este trabajo, ponemos en práctica estas alternativas y recomendamos el uso de un set de tasas de descuento decrecientes en el tiempo, con algunos ajustes.

\section{Metodología}

En esta sección se exponen los detalles de la derivación matemática de la tasa social de descuento a partir del modelo de estimación gamma. Además, se describen las técnicas de recolección de datos y se expone el proceso de ajuste por sesgo de valor presente, propuesto para las estimaciones en un contexto de corto plazo.

\section{Descuento gamma}

A partir de los argumentos expuestos que respaldan el uso de una tasa de descuento decreciente en el tiempo, en este trabajo se adapta la metodología de descuento gamma propuesta por Weitzman (2001) y la extensión propuesta por Edwards (2016), para estimar un set de tasas de descuento para el Ecuador. Una de las ventajas de esta metodología es que en ella se reconocen la dificultad y la incertidumbre existentes al definir una tasa social de descuento y, consecuentemente, se propone un mecanismo simple que integra la percepción de un grupo de expertos en un solo factor de descuento. Adicionalmente, el modelo demuestra que al agregar la opinión de un grupo de individuos se converge a una estructura de tasas de descuento decrecientes en el tiempo, incluso cuando a nivel individual cada sujeto se identifica con un mecanismo de descuento exponencial, típicamente empleado en la práctica.

El modelo parte de dos argumentos básicos. El primero es que no existe, al menos en lo referente al corto plazo, un consenso sobre el valor de la tasa social de descuento que debería aplicarse en proyectos con beneficios y costos en el largo plazo (por ejemplo, más de 20 años y 50 años). En la práctica, los economistas usan varias formas para determinar un nivel aproximado, por ejemplo, hacerlo mediante la productividad marginal del capital, la elasticidad del consumo o incluso la estimación de las preferencias individuales sobre el tiempo. Cada método prioriza un aspecto específico del análisis 
de la inversión pública; la equidad intergeneracional, el cambio tecnológico, las distorsiones de la economía, los efectos en el medio ambiente y la presencia de externalidades, entre otros, son todos temas de interés (Correa, 2008a). El segundo argumento sostiene que para integrar la heterogeneidad de opiniones y tipos de personas que son consultadas, capturada en una distribución de probabilidad, se deben promediar las funciones de descuento y no las tasas informadas. Para entender este supuesto, tomamos el ejemplo de Edwards (2002). Supóngase que dos individuos sensatos y perfectamente informados consideran que la TSD debe ser del 10\% y el 7\%, respectivamente. Si se busca ponderar ambas opiniones por igual, entonces el valor presente de un dólar dentro de 15 años estará dado por $0,5(1 / 1,10)^{15}+0,5(1 / 1,07)^{15}=0,3001$; no obstante, este valor será menor si lo que se pondera son directamente las tasas de descuento informadas $(1 / 1,085)^{15}=0,2941$.

Otro supuesto del modelo es que representa la estructura de descuento individual mediante el modelo estándar ${ }^{2}$. Es decir, se asume que individualmente las personas asignan ponderaciones en el tiempo a través de un arreglo exponencial $D=e^{-r t}$, donde la tasa de descuento informada viene dada por $\frac{D^{\prime}}{D}$ y es igual al parámetro $r$ para todos los retrasos de tiempo $t^{3}$. En términos prácticos, aunque gran cantidad de información experimental sugiere tasas individuales de descuento decrecientes en el tiempo (véanse, por ejemplo, Mazur, 1987; Benhabib, Bisin y Schotter, 2010; Castillo y Zhangallimbay, 2018), las aplicaciones más comunes consideran una función exponencial como modelo de análisis; por lo tanto, es fácil suponer que las opiniones obtenidas parten de este modelo de referencia. Finalmente, el modelo incorpora lo que el autor define como "incertidumbre irreducible", suponiendo que las tasas sugeridas (opiniones) siguen una distribución de probabilidad gamma. En este caso, la función decreciente de la tasa de descuento se genera por la incertidumbre individual respecto del valor que puede tomar la tasa en períodos distantes de tiempo y no necesariamente por una modalidad de preferencias individuales distinta a la tradicional, como, por ejemplo, el modelo hiperbólico.

Para verlo de manera sencilla, imaginemos a un ejecutivo encargado de analizar un proyecto de relevancia social para una institución internacional de desarrollo. La incertidumbre en lo referente a la decisión de aceptar o no el proyecto lleva al ejecutivo a considerar que la mejor manera de evaluar su factibilidad económica es mediante la opinión y la colaboración de un grupo de expertos, que están encargados de proponer un mecanismo de descuento ideal para transformar las unidades monetarias en términos equivalentes. El problema general se representa de la siguiente forma:

$$
\int_{0}^{\infty} A(t) Z(t) d t
$$

Donde $Z(t)$ es el beneficio neto y $A(t)$ el factor de descuento integrado en cada período $t$. Si los flujos $Z(t)$ vienen dados, entonces el factor $A(t)$ debe ser el resultado de considerar las $j$ opiniones aportadas por los expertos $A_{j t}$. El ejecutivo responsable de tomar la decisión sabe que debe considerar la opinión de todos los expertos antes de determinar una tasa social de descuento; sin embargo, no existe una respuesta única sobre cómo hacerlo. Aquí es donde los supuestos intervienen. La estructura exponencial $A_{j t}=e^{-x_{t} t}$ para todo $j=1,2, \ldots, n$ permite modelar el proceso a partir del cual se generan las opiniones y las respectivas tasas de descuento $x_{j}$. Adicionalmente, el supuesto de distribución gamma determina la probabilidad de que $x_{j}$ (opiniones) tome un determinado valor $x$ a través de la siguiente función:

$$
f(x)=\frac{\beta^{\alpha}}{\Gamma(\alpha)} x^{\alpha-1} e^{-\beta x}
$$

\footnotetext{
2 Weitzman (2001) concluye que, aunque todas las personas consultadas afirmen que la tasa de descuento es constante, la tasa de descuento efectiva declina fuertemente en el tiempo.

$3 D^{\prime}$ representa la primera derivada de la función de descuento exponencial D.
} 
Donde el factor de descuento $A(t)$ es el promedio ponderado del factor o función de descuento de cada integrante del grupo:

$$
A(t)=\frac{\beta^{\alpha}}{\Gamma(\alpha)} \int_{0}^{\infty} x^{\alpha-1} e^{-(\beta+t) x} d x
$$

$\alpha$ y $\beta$ son parámetros de la función gamma que se estiman a través de los datos. La tasa de descuento $R_{t}$ implícita viene dada por:

$$
R(t)=-\frac{A^{\prime}(t)}{A(t)}
$$

Donde $A^{\prime}(t)$ es la primera derivada del factor de descuento integrado $A(t)$. Al resolver (4) y (5), se obtiene la tasa social de descuento:

$$
R(t)=-\left(\frac{\beta}{\beta+t}\right)^{\alpha}
$$

Mediante la ecuación (6), se puede deducir fácilmente que una estructura decreciente de tasa de descuento es el resultado de agregar o integrar las diversas opiniones, a través de la distribución de probabilidad gamma. Esta caracterización del descuento es compatible con varias teorías del comportamiento que sugieren la existencia de preferencias inconsistentes a nivel agregado (tasas decrecientes), incluso cuando a nivel individual las preferencias se muestran consistentes (tasas constantes). Una de las ventajas de aplicar la distribución de probabilidad gamma es que sus parámetros $\alpha$ y $\beta$ pueden ser expresados en términos de la media muestral $(\mu)$ y la desviación estándar $\sigma$ de los datos:

$$
\begin{aligned}
& \alpha=\frac{\mu^{2}}{\sigma^{2}} \\
& \beta=\frac{\mu}{\sigma^{2}}
\end{aligned}
$$

Si se reemplazan (7) y (8) en la ecuación (6), se obtiene la tasa social de descuento efectiva en términos de la media y la varianza de la muestra.

$$
R(t) \equiv \frac{\mu}{1+\frac{\sigma^{2}}{\mu} t}
$$

Finalmente, la ecuación (9) indica una relación inversa entre la tasa social de descuento y la medida de incertidumbre $\sigma$ de la muestra. Además, la función considera el tiempo como una variable continua. Para manejar la TSD desde un punto de vista discreto, Weitzman (2001) determina el promedio para cinco intervalos definidos de tiempo: futuro inmediato (del año 1 al año 5), promedio del 4\%; futuro cercano (del año 6 al año 25), promedio del 3\%; futuro medio (del año 26 al año 75), promedio del 2\%; futuro lejano (del año 76 al año 300), promedio del 1\%, y futuro muy lejano (del año 300 en adelante), promedio del 0\%. En el presente caso de aplicación al Ecuador, considerando algunos criterios operativos de la evaluación de proyectos de inversión pública que se llevan adelante, se evalúan las tasas en los intervalos de tiempo que se indican en el cuadro 2. 


\section{Cuadro 2}

Horizontes de evaluación

\begin{tabular}{cl}
\hline Intervalo (años) & \multicolumn{1}{c}{ Horizonte de evaluación } \\
\hline $0-5$ & Futuro inmediato \\
\hline $6-20$ & Futuro mediano \\
\hline $21-50$ & Futuro lejano \\
\hline $51-100$ & Futuro muy lejano \\
\hline
\end{tabular}

Fuente: Elaboración propia.

Nota: Intervalos de tiempo establecidos en el análisis.

La estimación de la TSD por el método de estimación gamma supone un enfoque de descuento distinto a los enfoques anteriores (Harberger, 1972); el resultado es un parámetro de descuento decreciente según el horizonte de evaluación de un proyecto de inversión. Asimismo, su aplicación pone énfasis en el análisis del costo de oportunidad intertemporal de los recursos, por lo que la TSD resultante no se ve afectada por cambios en los demás precios económicos - precios cuenta o de eficiencia-, ni tampoco los afecta.

En aproximaciones anteriores a la estimación de la TSD constante se emplean como referencia, entre otras alternativas, estimaciones de la productividad media del capital, basada en proyectos marginalmente aceptados y cuyo proceso de evaluación económica se realizó a partir del ajuste de los precios de mercado a precios de eficiencia, para el cálculo de la tasa interna de retorno económico. Esta práctica tiene varias limitaciones. Por un lado, en los proyectos aprobados se tiende a sobreestimar los flujos de beneficios, por lo que la productividad media del capital puede constituir únicamente una referencia al límite superior o tasa máxima de descuento. Por otro lado, las limitaciones de información son importantes; no solo es difícil identificar una muestra representativa de proyectos de inversión evaluados a precios de eficiencia, sino que también es típico que la actualización de los precios de eficiencia tenga rezagos importantes y las oficinas estatales de planificación o los bancos de desarrollo difícilmente promueven procesos de revisión. A esto se suman dinámicas de integración regional que han reducido las distorsiones que motivan los ajustes en los factores de conversión de los precios de mercado, por ejemplo, políticas de reducción de aranceles, eliminación de subsidios o reducción de impuestos, entre otras.

El método gamma aplicado en este estudio se distancia de esta problemática por cuanto la información de referencia se deriva de un instrumento de decisión independiente, aplicado a personas responsables de la toma de decisiones. La percepción captada mediante dicho instrumento está fundada en la experiencia en evaluación de proyectos de quienes participaron en el estudio, por lo que a priori puede afirmarse que su decisión debe resumir todos los elementos de análisis expuestos, independientemente de la necesidad de actualización de los precios de eficiencia de los factores para la evaluación económica de proyectos de inversión.

\section{Datos y adaptación}

Para la aplicación del método propuesto, se trabajó con una encuesta aplicada a un grupo de expertos, analistas y responsables de la toma de decisiones que trabajan en temas relacionados con el análisis de inversiones y la evaluación de proyectos de inversión pública y privada. El grupo incluyó cuatro categorías de personas: docentes de las facultades de economía y administración de diversas universidades (académicos), estudiantes de posgrado en las áreas de economía y negocios (maestrantes), gerentes y analistas del sector financiero vinculados a la asociación de bancos privados del país (financieros) y gerentes o empresarios vinculados a las cámaras de industria, producción y comercio (empresarios) ${ }^{4}$.

\footnotetext{
4 En este aspecto, los autores agradecen la colaboración de la Asociación de Bancos Privados del Ecuador (Asobanca) y la Cámara de Industrias y Producción (CIP).
} 
En el proceso de levantamiento de información se enviaron 950 encuestas mediante correo electrónico. Las encuestas se diseñaron y distribuyeron mediante la herramienta digital SurveyMonkey. Asimismo, se realizaron gestiones de confirmación de la recepción de los correos electrónicos y presentación del estudio a los participantes, directamente y a través de las asociaciones de las que son miembros. La tasa de respuesta fue del $28 \%$. Se obtuvieron 269 encuestas completas, distribuidas entre los cuatro grupos de referencia del estudio ${ }^{5}$. En el cuadro 3 se presenta la composición de la muestra.

Cuadro 3

Composición de la muestra de personas que respondieron la encuesta diseñada para el estudio

\begin{tabular}{lrc}
\hline Categoría & Frecuencia & Porcentaje \\
\hline Académicos & 167 & 62,08 \\
\hline Maestrantes & 82 & 30,48 \\
\hline Financieros & 5 & 1,86 \\
\hline Empresarios & 11 & 4,08 \\
\hline Total & 269 & 100 \\
\hline
\end{tabular}

Fuente: Elaboración propia.

Nota: La encuesta se aplicó de forma digital mediante la herramienta SurveyMonkey.

Weitzman (2001) consultó directamente a los encuestados por el parámetro de TSD para descontar los beneficios y los costos de proyectos de mitigación del cambio climático ${ }^{6}$. La pregunta que planteó en su estudio es: teniendo en cuenta todas las consideraciones relevantes, ¿qué tasa de interés real cree que debería usarse para descontar en el tiempo los beneficios (esperados) y los costos (esperados) de los proyectos propuestos para mitigar los posibles efectos del cambio climático global?

Sin embargo, este proceso presenta varios inconvenientes. En algunos casos, los encuestados afirman que no cuentan con el conocimiento suficiente en este ámbito para emitir una opinión; otros manifiestan la necesidad de tener tasas diferenciadas para países desarrollados y países en desarrollo; finalmente, algunos ponen de manifiesto la incomprensión general de la pregunta. Aun en grupos de personas experimentadas es posible encontrar problemas de comprensión, lo que puede traducirse en sesgos en las respuestas y estimaciones respectivas. No obstante, las diferencias de opiniones y juicios de valor respecto de los mecanismos de descuento tienen orígenes más fundamentales en relación con el bienestar intergeneracional y la asignación de recursos públicos, entre otros aspectos (Weitzman, 2001). Para minimizar el factor de confusión, Edwards (2016) propone una modalidad diferente de encuesta, en que se emplean tres preguntas hipotéticas de fácil comprensión y cuya estructura de recolección es adaptativa, es decir, condicionada según las respuestas previas. De esta forma, en lugar de obtenerse un parámetro único, se obtiene un rango de descuento de cada uno de los encuestados (véase el anexo A1) ${ }^{7}$.

La encuesta empleada en el presente estudio consiste en tres preguntas de elección entre proyectos de inversión con beneficios en el largo plazo. Para capturar el potencial sesgo de diseño en la magnitud del parámetro, se definieron tres versiones del formulario (E1, E2 y E3) (véanse, por ejemplo, Coller y Williams, 1999; Harrison y otros, 2005). Cada versión difiere en los montos generados por los proyectos de inversión: montos bajos, montos medios y montos altos (véase el anexo A2) ${ }^{8}$.

5 De las respuestas recibidas, destacamos su calidad, así como la coherencia observada entre las respuestas y las preguntas de control.

6 La muestra de este estudio asciende a alrededor de 2.100 observaciones, incluidos expertos y economistas de todo el mundo.

7 Las preguntas apuntan a elegir entre dos posibles proyectos de inversión: uno que genera beneficios valorados en 1,5 millones de dólares en 15 años y otro que genera 2,5 millones de dólares en 30 años.

8 Si bien este proceso no corrige completamente el problema de encuadre (framing), permite obtener una mayor variación de las tasas de descuento aproximadas por la encuesta, capturando un mayor rango de preferencias sobre el descuento. 
Asimismo, cada versión permite definir cuatro rangos de descuento: a medida que aumentan los montos, los rangos de descuento también difieren; por lo tanto, se dispone en total de 12 rangos de descuento (véase el cuadro 4). Por razones de simplicidad y eficiencia, se toma el punto medio de cada rango como referencia aproximada de la tasa social de descuento para cada encuestado ${ }^{9}$. El rango general de las 12 opciones va desde 0,98\% hasta 14,13\%. Nótese también que el rango incluye la tasa administrativa actual del 12\%, como un mecanismo de validación.

\section{Cuadro 4}

Rangos de descuento

(En porcentajes)

\begin{tabular}{lccc}
\hline Encuesta & Límite inferior & Límite superior & $\begin{array}{c}\text { Tasa social de descuento } \\
\text { (TSD) aproximada }\end{array}$ \\
\hline E1 & 0 & 1,96 & 0,98 \\
\hline E1 & 1,96 & 3,41 & 2,69 \\
\hline E1 & 3,41 & 6,54 & 4,98 \\
\hline E1 & 6,54 & - & 6,54 \\
\hline E2 & 0 & 4,62 & 2,31 \\
\hline E2 & 4,62 & 7,32 & 5,97 \\
\hline E2 & 7,32 & 10,73 & 9,03 \\
\hline E2 & 10,73 & - & 10,73 \\
\hline E3 & 0 & 3,41 & 1,70 \\
\hline E3 & 3,41 & 10,73 & 7,07 \\
\hline E3 & 10,73 & 14,13 & 12,43 \\
\hline E3 & 14,13 & - & 14,13 \\
\hline
\end{tabular}

Fuente: Elaboración propia.

Nota: Las encuestas se distribuyen de manera independiente.

Finalmente, se recogen también algunas características individuales de los participantes, para evaluar su posible relación con los niveles de descuento, entre ellas, edad, género, experiencia laboral, número de hijos, aversión al riesgo y otras (véase el cuadro 5).

Cuadro 5

Características de la muestra

\begin{tabular}{lccrrr}
\hline & $N$ & Media/proporción & $\begin{array}{c}\text { Desviación } \\
\text { estándar }\end{array}$ & Mínimo & Máximo \\
\hline Mujer & 199 & 0,32 & 0,47 & 0 & 1 \\
\hline Nivel de educación & 200 & 3,12 & 0,55 & 2 & 4 \\
\hline Edad & 199 & 38,28 & 8,90 & 23 & 63 \\
\hline Tiene hijos & 200 & 0,61 & 0,49 & 0 & 1 \\
\hline Experiencia laboral (años) & 200 & 14,94 & 8,59 & 2 & 43 \\
\hline Aversión al riesgo & 207 & 6,70 & 1,99 & 1 & 10 \\
\hline Riesgo (Dospert) & 207 & 4,98 & 1,43 & 1,33 & 9 \\
\hline
\end{tabular}

Fuente: Elaboración propia.

Nota: Algunos encuestados no respondieron la encuesta en su totalidad, por lo que existen divergencias en las observaciones. Las categorías para el nivel de educación son: 1= secundaria, 2= universitaria, 3= postgrado, 4= doctorado. La aversión al riesgo corresponde a una medida autodeclarada de disposición a asumir riesgo, en una escala de 1 a 10. Dospert corresponde al promedio de las medidas autodeclaradas de riesgo en seis dominios: conducción de un vehículo, finanzas, deportes, salud, área laboral y confianza en otras personas.

\footnotetext{
9 Edwards (2016) considera que, cuando se obtiene un rango de descuento, es necesario estimar la TSD; sin embargo, esta aproximación no representa un cambio significativo en la media y la varianza de la muestra necesarias en la estimación gamma, en comparación con la aplicación de una tasa aproximada por el punto medio del rango.
} 


\section{Ajuste por preferencias de corto plazo}

En la metodología de descuento gamma, el mecanismo de recolección de datos se expresa, por lo general, en un contexto de preferencias de largo plazo. Si bien un individuo con preferencias consistentes en relación con el tiempo debe mantener, en teoría, una misma estructura de descuento intertemporal en el corto y el largo plazo, el sesgo de valor presente, es decir, la tentación de obtener retornos inmediatos, cuando la consecución de estos es inminente, constituye una anomalía que produce distorsiones entre ambos enfoques. Debido a que la metodología de Edwards (2016) no captura adecuadamente las preferencias en períodos de corto plazo, ajustamos nuestros resultados incluyendo la siguiente pregunta en la encuesta: ¿Cuál es el monto mínimo que usted estaría dispuesto a recibir hoy (en dólares) en lugar de recibir 200 dólares dentro de tres meses?

Del valor informado se deriva una tasa de descuento efectiva ${ }^{10}$ determinada en un período de retraso de tres meses. Aplicando equivalencia de tasas, se obtiene un valor para la tasa efectiva anual de cada encuestado. Con el mismo mecanismo de integración de opiniones, se puede estimar una función general de tasa de descuento decreciente sobre la base del retraso $t$ (años). La pregunta es si este ejercicio refleja un comportamiento similar al observado en la función integrada de descuento en el mecanismo original de largo plazo. Si bien el mecanismo original propone un contexto de elección de proyectos de inversión con retornos en períodos distantes en el tiempo, el mecanismo no es inocuo a las preferencias individuales. Por lo tanto, si existen diferencias sustanciales en las tasas determinadas por cada una de las funciones, en los distintos intervalos de tiempo establecidos, una mejor aproximación a la TSD debe ser un promedio de las tasas resultantes.

\section{Resultados}

En primer lugar, se analizan los resultados obtenidos mediante el mecanismo original de encuesta en un enfoque de largo plazo. En el gráfico 1 se presenta el histograma de frecuencia de las tasas aproximadas de descuento, recogidas y obtenidas mediante las tres preguntas de la encuesta, en sus tres versiones aplicadas a los expertos, académicos, maestrantes, financieros y empresarios. A través de la metodología de descuento gamma, se integraron las preferencias y se estimó una función decreciente de la TSD.

La ecuación (9) sugiere que la tasa social de descuento es igual al promedio ponderado de los datos $\mu$ cuando $t=0$; en el presente caso, esta tasa asciende al 7,17\%. Según el mecanismo de estimación, $t=0$ corresponde a los períodos inferiores al período mínimo incluido en la encuesta, que es de 15 años. La tasa instantánea en nuestros resultados es mayor que el valor del 3,96\% obtenido por Weitzman (2001) y que el valor del 4,92\% obtenido por Edwards (2016) en el caso de Chile, pero menor que el valor del 10,76\% encontrado por Correa (2008b) en el caso de Colombia. La desviación estándar $\sigma$ de nuestros datos es 3,0024, menor que la encontrada en Colombia (Correa, 2008b) y aproximada a la de Weitzman (2001). Reemplazando los parámetros $\mu$ y $\sigma$, la tasa efectiva de descuento para cada período $t$, según el método de Weitzman (2001), está dada por:

$$
R(t)=\frac{7,1722}{1+\frac{(3,0024)^{2}}{7,1722}}
$$

\footnotetext{
${ }^{10} \mathrm{Al}$ aplicar el modelo exponencial, el monto (valor presente) informado por los participantes es igual a 200 $e_{-r t}$, donde la tasa de descuento individual viene dada por $r$.
} 


\section{Gráfico 1}

Histograma de la tasa social de descuento (TSD)

(En porcentajes)

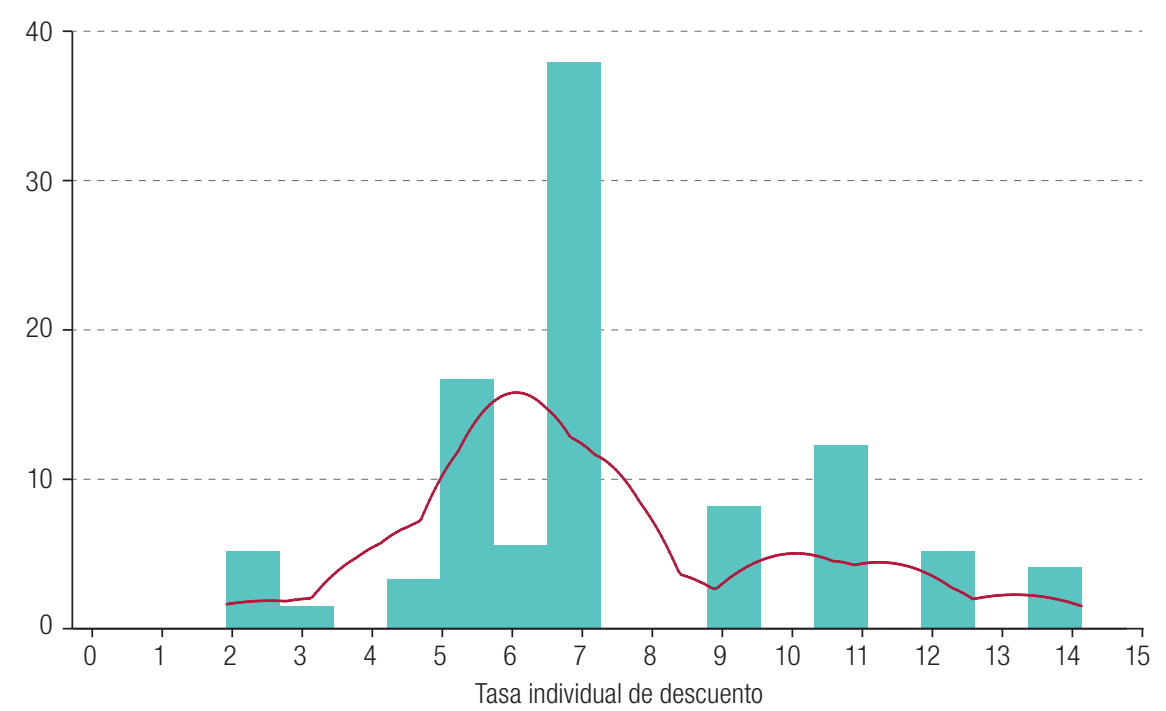

Fuente: Elaboración propia, sobre la base de la tasa social de descuento obtenida mediante una encuesta de tres preguntas.

Un elemento relevante es que en períodos de corto plazo, por ejemplo, menores que 15 años, la metodología propuesta tiene dificultades para reflejar las preferencias sociales ${ }^{11}$. No obstante, por razones prácticas, en las estimaciones de este estudio se asume un comportamiento consistente desde los primeros años. De esta forma, para evaluar proyectos de inversión pública en el Ecuador, el flujo económico generado por la inversión en el año 5 se debe descontar a una tasa del 6,87\%, mientras que los flujos generados en los años 15 y 30 se deben descontar a tasas del 6,03\% y el $5,21 \%$, respectivamente. Nótese que se estima una sola tasa para cada año de retraso. Sin embargo, es posible determinar una tasa constante y equivalente $(\bar{R})$ al proceso decreciente de la tasa de descuento mediante la ecuación (12).

$$
\bar{R}=\frac{1}{\int_{0}^{\infty} A(t) d t}
$$

Reemplazando (3) en (9), se obtiene:

$$
\bar{R}=\frac{(\mu-\sigma)(\mu+\sigma)}{\mu}
$$

La tasa constante equivalente para nuestras estimaciones se ubica en un 5,91\% anual, valor considerablemente menor que el nivel de la tasa administrativa aplicada por varios países y algunas organizaciones multilaterales. Respecto a otras estimaciones similares en la región, la tasa equivalente $\bar{R}$ resulta menor que la obtenida en Colombia (8,0\%) y mayor que la estimada en Weitzman (2001) (1,43\%).

En el gráfico 2A se muestra la dinámica de la tasa social de descuento integrada. Se puede observar un decrecimiento relativamente moderado, al pasarse de una tasa de alrededor del $7 \%$ para el primer año a una de aproximadamente el 3\% para el año 100.

\footnotetext{
${ }^{11}$ Edwards (2016) reconoce que las tasas que pueden inferirse a través de su metodología solo son consistentes si se refiere a un año posterior al año inicial establecido en la encuesta, que en nuestro caso es el año 15.
} 


\section{Gráfico 2}

Tasa social de descuento (TSD) estimada para el largo plazo

(En porcentajes)

A. TSD decreciente continua

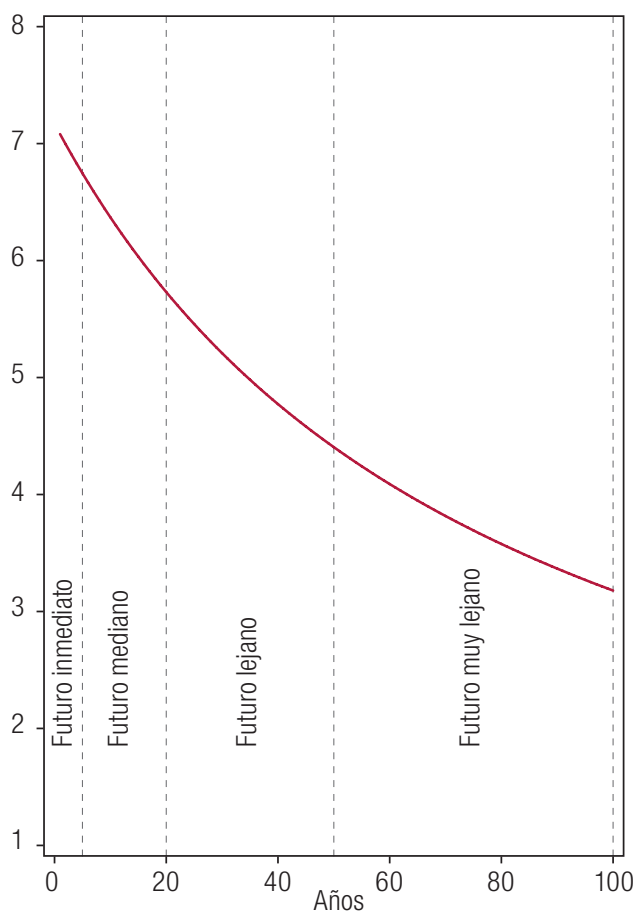

B. TSD decreciente por intervalos

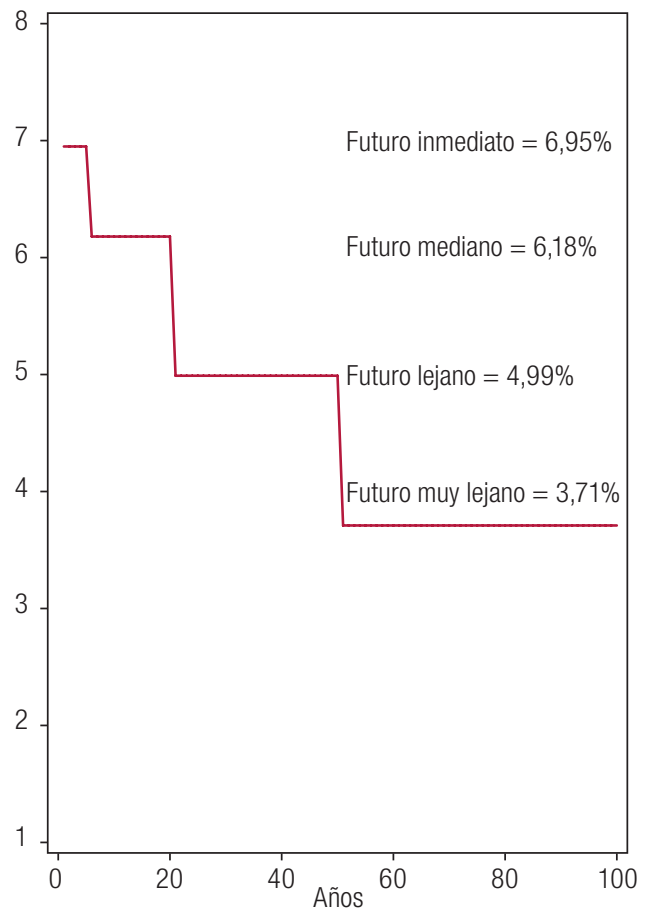

Fuente: Elaboración propia, sobre la base del mecanismo de estimación de preferencias (encuesta).

Para implementar una modalidad de descuento decreciente, recogiendo así en los criterios de evaluación el amplio conjunto de información que respalda esta dinámica de descuento, se pueden considerar los intervalos de tiempo definidos previamente. En cada segmento se agrupan los valores de la TSD sobre la base del promedio de la función en el intervalo de tiempo correspondiente al horizonte de evaluación. En el gráfico 2 se muestra la dinámica de la TSD para el caso ecuatoriano. Los resultados preliminares sugieren un descuento de alrededor del 7,0\% para los flujos de los primeros 5 años, del 6,0\% para los siguientes 15 años, del 5,0\% para los siguientes 30 años y del 3,7\% para los siguientes 50 años. Para evaluaciones de proyectos con efectos más extensos, cuyo horizonte supere los 100 años, se puede aplicar una tasa del 1,0\% (véase el gráfico 2B). Si bien en la práctica estos horizontes de evaluación no son comúnmente empleados, menos aún en la región, en los Estados Unidos, por ejemplo, la Agencia de Protección Ambiental (EPA) considera horizontes de más de 100 años para analizar sus múltiples programas de investigación, entre ellos, el programa sobre aire, clima y energía, el programa de investigación sobre seguridad química para la sostenibilidad y el programa de investigación sobre recursos hídricos seguros y sostenibles.

El supuesto básico de las estimaciones es que, por su estructura, la encuesta obliga a los sujetos a considerar opciones de beneficios para futuras generaciones, con lo que las preferencias de beneficios para su propia generación (corto plazo) están subestimadas. Para corregir las potenciales inconsistencias en las estimaciones de las tasas de corto plazo, esto es, futuro inmediato y futuro mediano, se analiza la pregunta de preferencias individuales sobre el tiempo para un período de tres meses y con un valor futuro de 200 dólares. La información recolectada con la pregunta no tiene un rango definido previamente en el diseño de la encuesta y, a diferencia de Edwards (2016), se evita añadir el contexto hipotético de proyectos de inversión pública, obteniendo directamente las preferencias individuales sobre el tiempo. 
Los resultados muestran tasas significativamente más altas que las determinadas mediante el mecanismo inicial de Weitzman (2001). El rango va desde el 1\% hasta tasas superiores al 200\%. Este resultado se deriva potencialmente de dos fuentes: i) la magnitud de la recompensa hipotética de la pregunta, que artificialmente hace más atractivo el presente, o ii) el sesgo puro de valor presente (independiente del monto propuesto). Aun así, todavía es posible asumir que las preferencias individuales respecto de la tasa de descuento siguen una distribución de probabilidad gamma (véase el gráfico 3). Por lo tanto, se agrega la opinión de los expertos, se determina la función de descuento agregada y se estima la función para la TSD para los retrasos de $t$. Los resultados muestran tasas de descuento del $14 \%$, el $2 \%$, el $1 \%$ y el $0 \%$ para el futuro inmediato, el futuro mediano, el futuro lejano y el futuro muy lejano, respectivamente (véase al gráfico 4).

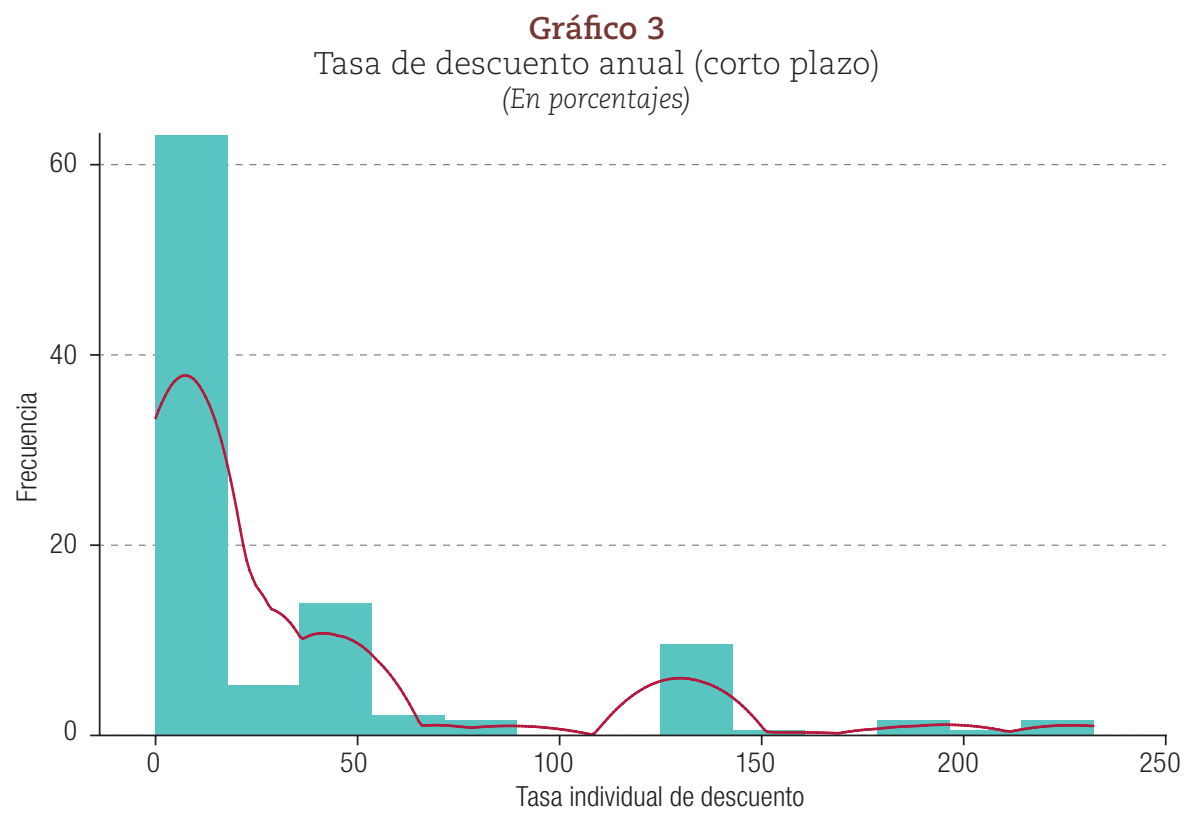

Fuente: Elaboración propia, sobre la base del mecanismo de estimación de preferencias (encuesta).

En el gráfico 4A se muestra la dinámica de las tasas anuales estimadas con el mecanismo de estimación de corto plazo. A diferencia de lo que se observa en el gráfico 2, el proceso muestra un decrecimiento más acelerado que en el contexto inicial (largo plazo), posiblemente derivado del aumento de la desviación estándar $(\sigma)$ de los datos en este mecanismo. Si se consideran ambos efectos, el de magnitud y el de varianza de la respuesta, lo que se esperaría observar en cuanto a las tasas estimadas en el contexto de corto plazo es una curva de decrecimiento más pronunciada, pero con tasas mayores que las del contexto de largo plazo. Sin embargo, nuestros resultados muestran un aumento considerable en los primeros años - por ejemplo, para descontar el flujo generado en el primer año, la función estimada sugiere una TSD superior al 120\% -, mientras que para el año 100 la TSD sugerida es menor que la del contexto de largo plazo, aproximadamente del $0 \%$. 


\section{Gráfico 4}

Tasa social de descuento (TSD) estimada para el corto plazo

(En porcentajes)

\section{A. TSD decreciente continua}

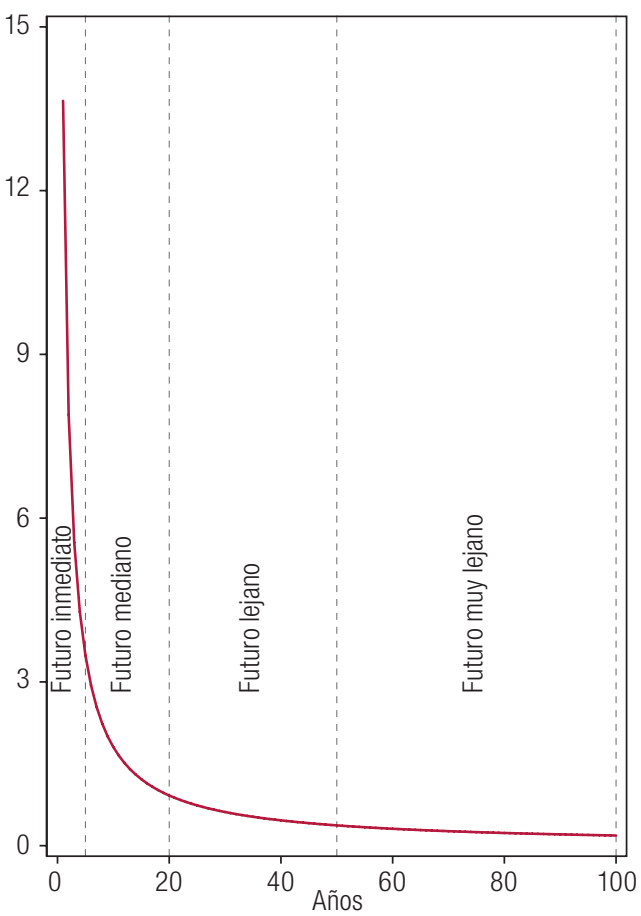

B. TSD decreciente por intervalos

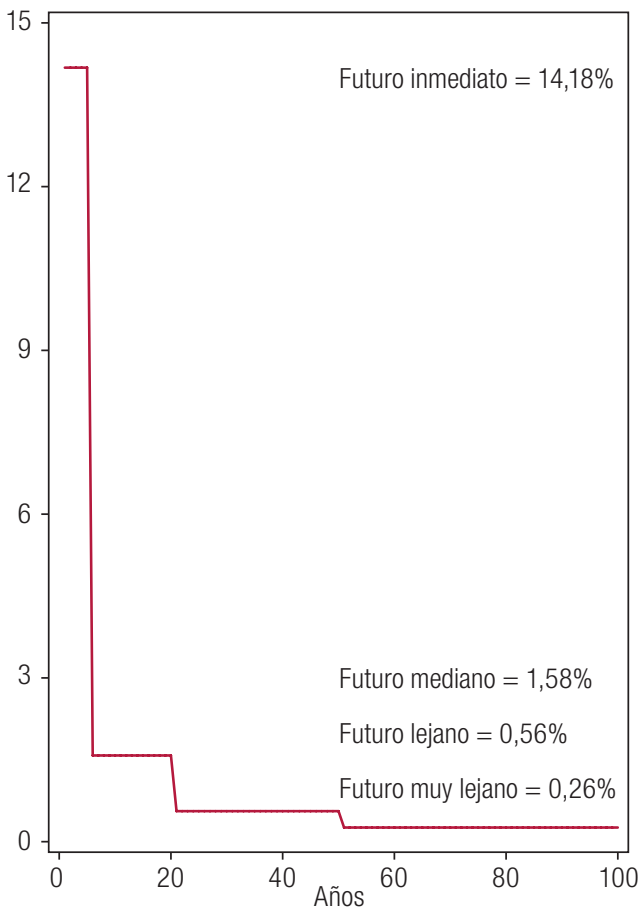

Fuente: Elaboración propia, sobre la base del mecanismo de estimación de preferencias (encuesta).

De manera similar a lo que ocurre en otros estudios (véanse Herrnstein, 1961; Ainslie y Herrnstein, 1981; Loewenstein y Prelec, 1992; Coller y Williams, 1999; Manzini y Mariotti, 2007; Benhabib, Bisin y Schotter, 2010; Castillo y Zhangallimbay, 2018), los resultados expuestos revelan las inconsistencias de las preferencias individuales respecto del tiempo y el contexto de análisis (véase el gráfico 5). Los mecanismos de obtención de preferencias, al igual que las alternativas de estimación de la TSD, no son universalmente consistentes y son sensibles a la modalidad de exposición o encuadre empleada en el formulario de recolección de información. Frente a esta disyuntiva instrumental, y tomando en cuenta que las alternativas planteadas son efectivas en relación con el costo frente a alternativas de obtención de mayor envergadura, se plantea la armonización de los sesgos implícitos en ambos contextos, dado que responden a la opinión y las preferencias sociales de los encuestados. Asimismo, es interesante observar que las tasas estimadas para el futuro inmediato (de 1 a 5 años) en ambos casos, corto y largo plazo, conforman un intervalo que va del $7 \%$ al $14 \%$, en el que quedan incluidas las tasas administrativas estándares usualmente empleadas. En otras palabras, nuestras estimaciones sugieren que estas tasas son buenos parámetros de evaluación para proyectos de corto plazo (menores que 5 años); sin embargo, para horizontes de evaluación mayores, estas tasas sobredevalúan los flujos netos futuros generados por un proyecto, en un proceso en que las preferencias por obtener retornos elevados en períodos cortos de tiempo predominan sobre la búsqueda de un equilibrio de beneficios que considere el efecto en futuras generaciones.

Para armonizar ambas aproximaciones y ajustar las estimaciones frente a los contextos de obtención de corto y de largo plazo, se evalúa el promedio de las funciones de ambas estimaciones. La línea continua del gráfico 5 muestra esta dinámica. Las elevadas tasas observadas en el contexto de corto plazo permiten ajustar el componente no considerado por la metodología de Edwards, 
precisamente lo que no permite establecer tasas de descuento adecuadas para los primeros años de vida de un proyecto. En este caso, para la corrección se consigue información de los mismos agentes, enfatizando la necesidad de obtener de manera más precisa el valor de los retornos de corto plazo, en especial de aquellos que potencialmente se recaudan en su misma generación.

\section{Gráfico 5}

Tasa social de descuento (TSD) ajustada para el corto y el largo plazo y su promedio (En porcentajes)

A. TSD decreciente continua

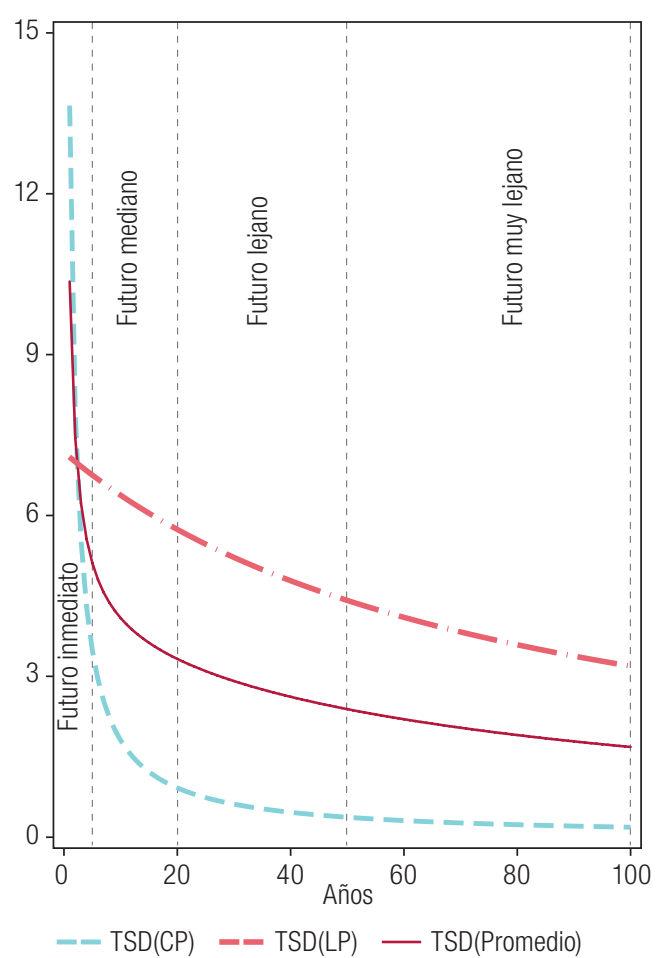

B. TSD decreciente por intervalos

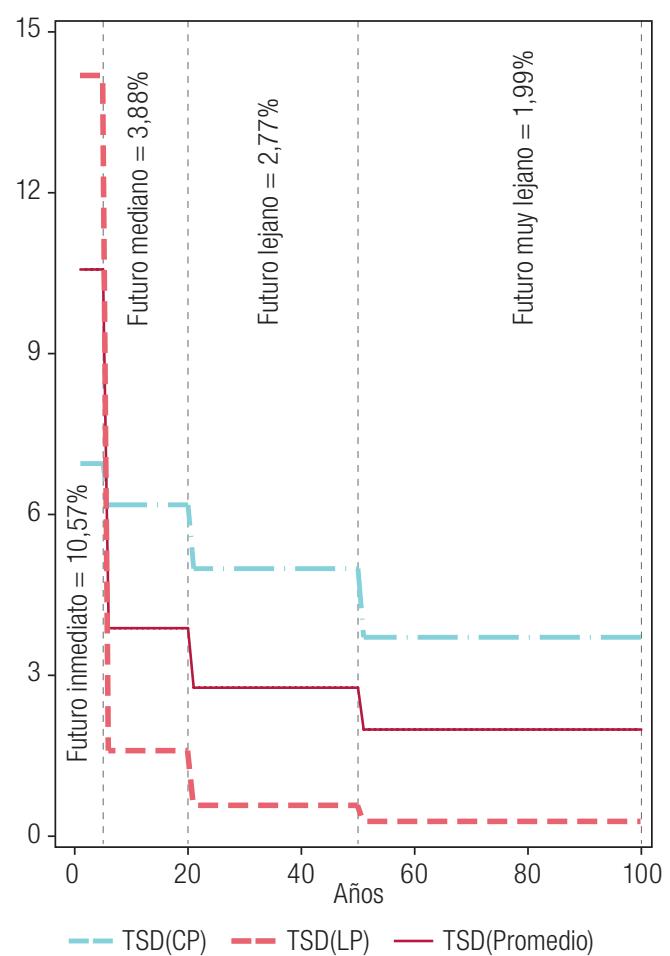

Fuente: Elaboración propia, sobre la base del mecanismo de estimación de preferencias (encuesta).

Considerando estos elementos de ajuste en nuestras estimaciones, los resultados sugieren la aplicación de una tasa de alrededor del 11\% para descontar los flujos económicos generados en el futuro inmediato (5 primeros años), de aproximadamente el $4 \%$ para los flujos generados en los siguientes 15 años, cercana al 3\% para los siguientes 30 años y del 2\% para los últimos 50 años, dentro de un horizonte de referencia de 100 años. Nuevamente, si el período de evaluación se extiende a más de 100 años, se recomienda emplear una tasa del 1\%.

\section{Determinantes de las preferencias de largo plazo}

Como un elemento adicional de análisis, que provee información relevante respecto del tipo de respuestas provistas por los expertos consultados, se analiza si existe una relación entre las características individuales y las preferencias informadas sobre el tiempo, específicamente en lo referente al largo plazo. El formulario de recolección de información recoge una batería de características individuales de los participantes, entre ellas el género, la edad, la experiencia laboral, el nivel de educación y el nivel de aversión al riesgo. Además, la estructura de la encuesta de tres preguntas empleada para obtener la TSD define cuatro intervalos de preferencias ordenados de forma ascendente y asignados en función 
de las respuestas de cada sujeto. Las dos versiones adicionales de la encuesta presentan un aumento de la magnitud de los beneficios de los proyectos de inversión pública; sin embargo, ofrecen otros intervalos de descuento con tasas distintas que son, en promedio, mayores. Mediante un análisis de regresión por intervalos, se evaluó la relación existente entre la tasa individual de descuento y las características de los participantes en el estudio.

En el cuadro 6 se presentan los resultados. Dos variables dicotómicas capturan el efecto fijo de las versiones adicionales de la encuesta (encuesta 2 y encuesta 3). Debido a que en promedio las tasas identificadas son mayores en las dos versiones adicionales, se encontró una relación positiva y significativa en una de estas variables. Es decir, existe un efecto de encuadre en la forma de obtener las tasas individuales de descuento, un elemento que se debe tomar en cuenta en el diseño de formularios. Además, se observa una relación positiva entre la edad y la TSD, si bien se trata de una relación débil (significativa solo al 10\% de significación estadística), que se manifiesta únicamente cuando la estimación incluye controles por preferencias individuales (aversión al riesgo). En términos del comportamiento, este resultado plantea que el comportamiento en lo referente al descuento cambia con la edad, una observación documentada en otras investigaciones; sin embargo, la relación observada es inversa a la constatación de otros estudios: cuanto mayor es la edad mayor es la tasa de descuento. Si bien en este resultado se pueden manifestar los niveles de impaciencia o autocontrol de los encuestados, debido a su baja significación se opta por no extraer más conclusiones. Finalmente, se encuentran datos empíricos que respaldan la relación entre las preferencias sobre el tiempo y el riesgo (significativos al 10\%), en el sentido de que individuos con más aversión al riesgo tienden a declarar su preferencia por mayores tasas de descuento (es decir, una mayor preferencia por el presente).

\section{Cuadro 6}

Determinantes de las preferencias sobre el tiempo

\begin{tabular}{|c|c|c|}
\hline & \multicolumn{2}{|c|}{$\begin{array}{c}\text { Variable dependiente } \\
\text { Intervalos de la tasa social de descuento (TSD) }\end{array}$} \\
\hline & (1) & (2) \\
\hline \multirow[t]{2}{*}{ Edad } & 0,4554 & $0,5091^{*}$ \\
\hline & $(0,2910)$ & $(0,2847)$ \\
\hline \multirow[t]{2}{*}{ Edad $^{2}$} & $-0,0048$ & $-0,0055^{\star}$ \\
\hline & $(0,0034)$ & $(0,0033)$ \\
\hline \multirow[t]{2}{*}{ Académicos } & $-0,6856$ & $-0,6813$ \\
\hline & $(0,8059)$ & $(0,7888)$ \\
\hline \multirow[t]{2}{*}{ Tiene hijos } & $-0,1323$ & $-0,0690$ \\
\hline & $(0,7607)$ & $(0,7481)$ \\
\hline \multirow[t]{2}{*}{ Mujer } & 0,2975 & 0,4265 \\
\hline & $(0,6508)$ & $(0,7208)$ \\
\hline \multirow[t]{2}{*}{ Experiencia laboral (años) } & $-0,0323$ & $-0,0251$ \\
\hline & $(0,0740)$ & $(0,0738)$ \\
\hline \multirow[t]{2}{*}{ Nivel de educación } & $-0,5960$ & $-0,5026$ \\
\hline & $(0,6027)$ & $(0,6221)$ \\
\hline \multirow[t]{2}{*}{ Aversión al riesgo } & & $0,3335^{\star}$ \\
\hline & & $(0,1896)$ \\
\hline \multirow[t]{2}{*}{ Riesgo (Dospert) } & & 0,0050 \\
\hline & & $(0,3056)$ \\
\hline \multirow[t]{2}{*}{ Encuesta 2} & $1,9580^{\star \star \star}$ & $1,9651^{\star \star \star}$ \\
\hline & $(0,7059)$ & $(0,6917)$ \\
\hline \multirow[t]{2}{*}{ Encuesta 3} & 1,4412 & 1,4008 \\
\hline & $(1,0633)$ & $(1,0515)$ \\
\hline
\end{tabular}




\begin{tabular}{lcc} 
Cuadro 6 (conclusión) & \multicolumn{2}{c}{ Variable dependiente } \\
& \multicolumn{3}{c}{ Intervalos de la tasa social de descuento (TSD) } \\
\cline { 2 - 3 } & $(1)$ & $(2)$ \\
\hline Constante & 0,6187 & $-3,1365$ \\
& $(5,7615)$ & $(6,2018)$ \\
\hline Otros controles & No & No \\
\hline Observaciones & 187 & 187
\end{tabular}

Fuente: Elaboración propia.

Nota: Se consideran los participantes de los que se cuenta con toda la información (187). La aversión al riesgo corresponde a una medida autodeclarada de disposición a asumir riesgo, en una escala de 1 a 10. Dospert corresponde al promedio de la aversión al riesgo autodeclarada en seis dominios (conducción de un vehículo, finanzas, deportes, salud, área laboral y confianza en otras personas).

Los errores estándares robustos se presentan entre paréntesis.

* Nivel de significación al 10\%; ** nivel de significación al 5\%; ${ }^{* \star \star}$ nivel de significación al 1\%.

\section{Conclusiones}

La tasa social de descuento constituye un parámetro fundamental para la evaluación económica y financiera de proyectos. Las decisiones del análisis de costo-beneficio, tanto en la evaluación de la inversión como en análisis de mayor envergadura, como los relacionados con el cambio climático, dependen de manera crucial de su nivel. No obstante, a pesar de su importancia, existe gran incertidumbre respecto de su nivel adecuado para cada país o contexto de análisis, ante lo cual en la mayoría de los casos se ha optado por una respuesta simple: emplear una tasa administrativa común. En el Ecuador, al igual que en otros países de la región, se emplea una tasa constante del 12\%; sin embargo, esta tasa dista mucho de incorporar la dinámica cambiante de las preferencias sociales sobre el tiempo.

En este documento se recoge la necesidad de actualización continua de los parámetros requeridos para la evaluación de la inversión pública. Se presenta una alternativa de estimación de la tasa social de descuento efectiva en relación con el costo que captura la abundante información empírica sobre el comportamiento respecto de las inconsistencias en el descuento temporal y el descuento decreciente en el tiempo (descuento hiperbólico), al mismo tiempo que ajusta las estimaciones en función de la diferencia de contexto en las decisiones de corto y largo plazo.

Los resultados, ajustados por el contexto temporal, sobre la base del promedio de las funciones de corto y largo plazo, arrojan un nivel distinto de descuento para cuatro horizontes de tiempo: futuro inmediato (aproximadamente 11\%), futuro mediano (aproximadamente 4\%), futuro lejano (aproximadamente $3 \%$ y futuro muy lejano (aproximadamente 2\%). En este contexto, las tasas administrativas comúnmente aplicadas se justifican en la evaluación de flujos de beneficios obtenidos en el futuro inmediato (5 años), lo que genera distorsiones importantes en la valoración económica de proyectos de inversión.

\section{Bibliografía}

Ainslie, G. y R. Herrnstein (1981), "Preference reversal and delayed reinforcement", Animal Learning Behavior, vol. 9.

Aldunate, E. y R. Martner (2006), "Política fiscal y protección social", Revista de la CEPAL, № 90 (LC/G.2323-P), Santiago, Comisión Económica para América Latina y el Caribe (CEPAL).

Benhabib, J., A. Bisin y A. Schotter (2010), "Present-bias, quasi-hyperbolic discounting, and fixed costs", Games and Economic Behavior, vol. 69.

Campos, J., T. Serebrisky y A. Suárez-Alemán (2016), Tasa de descuento social y evaluación de proyectos: algunas reflexiones prácticas para América Latina y el Caribe, Banco Interamericano de Desarrollo (BID). 
(2015), "Porque el tiempo pasa: evolución teórica y práctica en la determinación de la tasa social de descuento", Nota técnica del BID, N ${ }^{\circ}$ IDB-TN-861, septiembre.

Castillo, J. y D. Zhangallimbay (2018), "Las preferencias Individuales y sus determinantes: un análisis de las preferencias sobre el tiempo y el riesgo", Tesis para optar al grado de Magister en Ciencias Económicas, Guayaquil, Escuela Superior Politécnica del Litoral.

Comisión Europea (2008), Guide to Cost-Benefit Analysis of Investment Projects [en línea] https://ec.europa. eu/regional_policy/sources/docgener/guides/cost/guide2008_en.pdf.

Coller, M. y M. Williams (1999), "Eliciting individual discount rates", Experimental Economics, vol. 2.

Correa, F. (2008a), "Tasa de descuento ambiental Gamma: una aplicación para Colombia", Lecturas de Economía, vol. 69.

(2008b), "Un análisis teórico de las propuestas alternativas para la determinación de la tasa social de descuento de largo plazo", Semestre Económico, vol. 11.

Dasgupta, A. y D. Pearce (1972), Cost-Benefit Analysis: Theory and Practice, Palgrave Macmillan.

Delacámara, G. (2008), "Guía para decisores: análisis económico de externalidades ambientales", Documentos de Proyectos (LCM.200), Santiago, Comisión Económica para América Latina y el Caribe (CEPAL).

Edwards, G. (2002), "La tasa de descuento en proyectos de inversión de largo plazo", Revista de Análisis Económico, vol. 17, № 2.

Edwards, G. (2016), "Estimación de la tasa social de descuento a largo plazo en el marco de los sistemas nacionales de inversión: aplicación al caso chileno", El Trimestre Económico, vol. 329.

Fontaine, E. (2000), Evaluación Social de Proyectos, Alfaomega Editores.

Frederick, S., G. Loewenstein y E. D. O'Donoghue (2002), "Time discounting: a critical review", Journal of Economic Literature, vol. 40.

Harberger, A. (1972), "On measuring the social opportunity cost of public funds", Project Evaluation-Collected Papers, cap. 4, Chicago, The University of Chicago Press.

Harrison, G. y otros (2005), "Eliciting risk and time preferences using field experiments: some methodological issues", Research in Experimental Economics, vol. 10.

Herrnstein, R. (1961), "Relative and absolute strengths of response as a function of frequency of reinforcement", Journal of Experimental and Analysis Behavior, vol. 4.

Lowe, A. J. (2008), Intergenerational wealth transfers and social discounting: Supplementary Green Book guidance, HM Treasury.

Loewenstein, G. y D. Prelec (1992), "Anomalies in intertemporal choice: evidence and interpretation", Quarterly Journal of Economics, vol. 107.

Manzini, P. y M. Mariotti (2007), "Choice Over Time", IZA Discussion Paper, № 2993 [en línea] papers.ssrn. com/sol3/papers.cfm?abstract_id=1012547.

Mazur, J. (1987), "An adjusting procedure for studying delayed reinforcement", Quantitative Analyses of Behavior: The Effect of Delay and of Intervening Events on Reinforcement Value, vol. 5, M.L. Commons (eds.), Erlbaum, Hillsdale.

Ramsey, F. (1928), "A mathematical theory of saving", Economic Journal, vol. 38.

Thaler, R. (1981), "Some empirical evidence on dynamic inconsistency", Economic Letters, vol. 8.

Weitzman, M. (2001), "Gamma discounting", American Economic Review, vol. 91.

Zhuang, J. y otros (2007), "Theory and practice in the choice of social discount rate for cost-benefit analysis: a survey", ERD Working Paper, № 94, Banco Asiático de Desarrollo. 


\section{Anexo A1}

\section{Encuesta de tres preguntas}

1) Si usted tuviera que decidir hoy entre dos proyectos, un proyecto del Gobierno que genera beneficios que pueden ser valorados en 1,5 millones de dólares dentro de 15 años y un proyecto del Gobierno que genera beneficios que se pueden valorar en 2,5 millones de dólares dentro de 30 años, ¿cuál elegiría?

- $\quad$ 1,5 millones de dólares dentro de 15 años

- 2,5 millones de dólares dentro de 30 años

2) Usted eligió el proyecto que genera 1,5 millones de dólares dentro de 15 años sobre el proyecto que genera beneficios valorados en 2,5 millones de dólares dentro de 30 años.

Si ahora los beneficios de la segunda opción ascienden a 4,0 millones de dólares con el mismo horizonte de tiempo (30 años), ¿qué opción elegiría?

- $\quad$ 1,5 millones de dólares dentro de 15 años

- 4,0 millones de dólares dentro de 30 años

3) Usted eligió el proyecto que genera 2,5 millones de dólares en 30 años sobre la alternativa que genera beneficios por 1,5 millones de dólares en 15 años.

Si ahora los beneficios del proyecto a 30 años disminuyen a 2,0 millones de dólares, ¿qué opción elegiría?

- 1,5 millones de dólares dentro de 15 años

- 2,0 millones de dólares dentro de 30 años

\section{Estimación del intervalo de la tasa social de descuento (TSD)}

Por ejemplo, si una persona respondió que elige el proyecto que genera beneficios valorados en 2,5 millones de dólares dentro de 30 años sobre el proyecto que genera 1,5 millones de dólares en 15 años, se tiene entonces:

$$
\begin{aligned}
& 1,5 e^{-15 r}<2,5 e^{-30 r} \\
& \ln \left(1,5 e^{-15 r}\right)<\ln \left(2,5 e^{-30 r}\right) \\
& \ln (1,5)-15 r<\ln (2,5 e)-30 r \\
& r<3,41 \%
\end{aligned}
$$

Si este individuo respondió en la siguiente pregunta que elige el proyecto que genera 2,0 millones de dólares en 30 años sobre el que genera 1,5 millones en 15 años, se obtiene que:

$$
\begin{gathered}
1,5 e^{-15 r}<2,5 e^{-30 r} \\
r<1,96 \%
\end{gathered}
$$

Por lo tanto, la respuesta de este individuo, $r<1,96 \%$, se ubica en el primer rango de TSD propuesto. 


\section{Anexo A2}

\section{Versiones de la encuesta}

Cuadro A2.1

Montos de las encuestas

\begin{tabular}{lcc}
\hline Versión & Tiempo & $\begin{array}{c}\text { Monto } \\
\text { (en dólares) }\end{array}$ \\
\hline Encuesta 1 (montos bajos) & 15 años & 1,5 millones \\
\cline { 2 - 3 } & 30 años & 2,5 millones \\
\cline { 2 - 3 } & 30 años & 2,0 millones \\
\cline { 2 - 3 } & 30 años & 4,0 millones \\
\hline Encuesta 2 (montos medios) & 15 años & 5,0 millones \\
\cline { 2 - 3 } & 30 años & 15,0 millones \\
\hline Encuesta 3 (montos altos) & 30 años & 10,0 millones \\
\cline { 2 - 3 } & 30 años & 25,0 millones \\
\cline { 2 - 3 } & 15 años & 3,0 millones \\
\cline { 2 - 3 } & 30 años & 15,0 millones \\
\cline { 2 - 3 } & 30 años & 5,0 millones \\
\hline
\end{tabular}

Fuente: Elaboración propia.

Nota: Las encuestas se aplicaron de manera aleatoria. 